\title{
PRODUÇÃO CIENTÍFICA NOS PROGRAMAS DE PŐS-GRADUAÇÃO NAS ÁREAS DE INFORMAÇÃO NO BRASIL
}

\author{
SCIENTIFIC PRODUCTION IN THE POSTGRADUATE \\ PROGRAMS IN INFORMATION AREAS IN BRAZIL
}

\author{
Jonathan Rosa Moreiraa \\ Edberto Ferneda ${ }^{b}$
}

\begin{abstract}
RESUMO
Introdução: Compreender a produtividade científica dos Programas de Pós-Graduação no país pode favorecer o conhecimento sobre as diferentes áreas do saber. No caso das áreas de informação no Brasil, tal panorama pode apoiar estratégias, processos de tomada de decisão, além de evidenciar atividades de pesquisa, de modo a subsidiar políticas de fomento à pesquisa científica. Entretanto, ainda há espaços para estudos sobre como se comportam, ou quais são as características da produção científica dessas áreas em diferentes constructos, como nos programas de pós-graduação das respectivas áreas do conhecimento, por exemplo. Conhecer os fatores que induzem a produtividade nos programas de pós-graduação por meio de seus membros (pesquisadores e estudantes) pode apoiar estratégias e políticas de fomento e manutenção das atividades de pesquisa. Objetivo: O objetivo deste estudo é, portanto, descrever a produtividade científica dos programas brasileiros de pós-graduação nas áreas de informação (Arquivologia, Biblioteconomia, Ciência da Informação, Documentação e Museologia), representada por seus membros (pesquisadores e estudantes). Metologia: :Como estratégia metodológica, este estudo tem abordagem quantitativa, com uso da bibliometria como técnica quantitativa e estatística de medição dos índices de produção e disseminação do conhecimento científico. Resultados: Dos 20 Programas de Pós-Graduação estudados, 5 reúnem mais da metade do total de produção científica da área. A produção científica é divulgada, preferencialmente, em canais de comunicação de trabalhos completos em anais de congresso. Conclusão: Ainda no mesmo contexto, não há uma relação específica entre volume de pesquisadores e estudantes dos programas de pós-graduação e o volume da produção científica.
\end{abstract}

Descritores: Áreas de informação no Brasil. Produção científica. Produtividade

\footnotetext{
a Doutor em Ciência da Informação pela Universidade de Brasília (UnB). Pró-Reitor e Diretor Acadêmico da Educação Superior do Grupo Projeção e Diretor da Escola de Ciências da Saúde e da Vida. Email: jonathanmoreira@gmail.com.

b Doutor em Ciências da Comunicação (Ciência da Informação) pela Universidade de São Paulo Docente do Departamento de Ciência da Informação da Universidade Estadual Paulista (UNESP). Email: jonathanmoreira@gmail.com.
} 
científica. Programa de pós-graduação.

\section{INTRODUÇÃO}

Os estudos relacionados à pesquisa científica no Brasil, com enfoque na produtividade científica, podem revelar dados importantes para apoiar ações estratégicas que apoiem a comunidade científica das diferentes áreas do saber. No que se refere à produtividade científica, vários cenários podem ser analisados, dentre eles, os programas de pós-graduação. Ou seja, quando mais compreendermos padrões relacionados à produção científica, mais subsídios serão fornecidos às políticas de fomento e desenvolvimento nos programas de pós-graduação.

O tema em estudo trata da produção científica nas áreas de informação no Brasil (AIBR). Utiliza-se aqui o conceito de Vilan Filho (2010) que considera as AIBR como a reunião dos cursos de: Arquivologia, Biblioteconomia, Ciência da Informação, Documentação e Museologia. Para a CAPES, a área Ciência da Informação comporta os cursos de Arquivologia, Biblioteconomia e Documentação; considerando a Museologia como área distinta da Ciência da Informação. Então, para ajustar o conceito de AIBR aos interesses deste estudo, considera-se AIBR como a reunião das áreas de Ciência da Informação e Museologia.

O objetivo deste estudo foi descrever a produtividade científica dos programas brasileiros de pós-graduação nas AIBR (Arquivologia, Biblioteconomia, Ciência da Informação, Documentação e Museologia), representada por seus membros (pesquisadores e estudantes). Especificamente, busca apresentar (1) a produção científica dos membros dos programas brasileiros de pós-graduação nas AIBR; (2) apresentar a produção científica dos membros dos programas brasileiros de pós-graduação nas AIBR por canal de comunicação (artigos, livros, capítulos de livros, eventos, entre outros); (3) correlacionar a produção científica dos membros dos programas brasileiros de pós-graduação nas AIBR à quantidade de membros, quais sejam pesquisadores e estudantes; (4) correlacionar a produção científica dos membros dos programas brasileiros de pós-graduação nas AIBR à avaliação 
histórica do programa; e (5) apresentar a produção científica dos membros dos programas brasileiros de pós-graduação nas AIBR por distribuição geográfica.

Além de determinar as características típicas dos grupos de pesquisa das AIBR, bem como suas relações com a produção científica da área (MOREIRA, 2017), conhecer os fatores que induzem a produtividade nos Programas de PósGraduação (PPG) por meio de seus membros (pesquisadores e estudantes) pode apoiar estratégias e políticas de fomento e manutenção das atividades de pesquisa. Indiretamente, uma vez subsidiadas as políticas de fomento à pesquisa e desenvolvimento, tais resultados ainda podem favorecer os próprios PPG. Para além da compreensão de algumas características dos PPG, por meio de seus membros participantes de grupos de pesquisa, entender um pouco mais os grupos de pesquisa de uma determinada área, sobretudo no que se refere à sua produção científica, em termos de quantidade e canais de comunicação, é importante para testemunhar como esta área tem se comportado, se tem crescido, ou se precisa ser revitalizada (MOREIRA; VILAN FILHO; MUELLER, 2015). Cabe destacar que a produção científica é fulcral para o avanço da ciência (ALMEIDA; GUIMARÃES; ALVES, 2010) e a visibilidade científica legitimada pelos pares faz com que a comunidade científica interaja, proporcionando espaços para a construção de novos conhecimentos.

Ressaltam-se aqui os elementos que não são limitadores deste estudo, mas que são determinantes para a garantia da integridade dos resultados aqui alcançados. Um deles é que a veracidade dos dados está condicionada à veracidade das informações registradas pelos pesquisadores e estudantes em seus respectivos currículos Lattes. Por outro lado, a plataforma Lattes não delimita produção científica por PPG, ou seja, registra-se apenas a produção científica individual de participantes que podem constar em um ou mais PPG. A definição do conceito de produção científica do PPG pode então, para este estudo, ser considerada como o somatório da produção científica dos participantes (pesquisadores e estudantes) dos PPG. Pressupõe-se que toda produção científica individual de um determinado participante esteja contida na produção científica do PPG ao qual ele participa. No caso do participante pertencer a mais de um PPG, admite-se, então, dupla contagem. Corrobora-se, 
portanto, com o conceito de produção científica de Witter (1997), em um sentido mais lato, que a produção científica é uma expressão que engloba processos e produtos distintos, pessoas, associações, agências financiadoras e seus múltiplos consumidores. Neste sentido, há a materialização do conhecimento gerado em uma instituição de informação, contemplando todas as atividades acadêmicas e científicas de seus pesquisadores (SALOMÓN; RODRíGUEZ, 2007).

Sobre a sua estrutura, o artigo apresenta três macro seções: (i) referencial teórico; (ii) metodologia; e (iii) análise dos resultados e conclusões. No referencial teórico, são abordados conceitos e fundamentos da produção científica na perspectiva dos estudos bibliométricos, fazendo uma ilação com a metodologia do estudo, bem como o contexto da produtividade científica e suas relações sociais. A segunda seção apresenta a estratégia metodológica do estudo, com abordagem qualitativa e enfoque bibliométrico para tratamento dos dados coletados. A terceira seção apresenta os resultados com suas respectivas análises à luz do que os dados quantitativos revelam, assim como a base na literatura narrada no referencial teórico. $\mathrm{Na}$ terceira seção, portanto, observaremos o comportamento da produção científica nas áreas de informação no Brasil no contexto dos seus programas de pós-graduação, considerando diferentes variáveis, tais como canal de comunicação, volume de produção, quantidade de membros e conceito CAPES.

\section{PRODUÇÃO CIENTÍFICA E ESTUDOS BIBLIOMÉTRICOS}

Lourenço (1997, p. 52) diz que a produção científica "é toda a produção documental, independentemente do suporte (papel ou meio eletrônico), sobre um determinado assunto de interesse de uma comunidade científica específica". A importância da produção científica vem sendo objeto de estudos de muitos pesquisadores que partem de uma análise quantitativa sobre a forma como a mesma é comunicada e em que formato. Para Jankevictus (1995, p. 329), a pesquisa científica é a busca sistematizada de novos conhecimentos. Importante ressaltar que a produção cientifica nas IES é primordial para a relação dialógica entre as atividades de ensino, de pesquisa e de extensão. "As atividades de 
pesquisa são indispensáveis aos professores universitários, sem as quais seriam meros repassadores de informações de livrescas, de antemão ultrapassadas e que rapidamente se tornam inúteis com o avanço da fronteira do conhecimento".

A expressão "produção científica" é muito utilizada na literatura e no meio acadêmico. No entanto, é muito difícil defini-la com exatidão. O termo produção é utilizado em vários setores da vida econômica e social, expressando a criação ou realização de algo. No que se refere à produção científica, estudiosos da área observam que se refere sempre a um texto científico que tem coerência, consistência, originalidade e objetividade (ALVES, 2009, p. 104).

No Brasil, a pesquisa científica expandiu-se a partir da década de 60 , com a implantação e expansão dos cursos de pós-graduação, o que potencializou e acelerou a formação de recursos humanos especializados para as diversas áreas do conhecimento. Avaliar a produção científica de uma organização, instituições e grupos tem sido frequentemente foco de estudos. Entre os vários procedimentos usados para avaliar a ciência, destacam-se os estudos bibliométricos, que utilizam recursos da matemática e estatística, agora aplicados à mensuração da produção científica. (ALVES, 2009, p. 106).

Mugnaini, Jannuzzi e Quoniam (2004, p. 124) diz que os indicadores bibliométricos são úteis e importantes para se entender o ciclo de gestação, reprodução e disseminação da ciência e o aprimoramento da política científica e tecnológica nacional. Para o autor, transformar informação bibliográfica em indicadores bibliométricos não é uma tarefa simples, exigindo trabalho minucioso e cautela em cada passo (p. 130). O princípio da bibliometria é analisar a atividade científica ou técnica pelo estudo quantitativo das publicações, considerando a classificação e a frequência de produtividade científica, com tendências temáticas e metodológicas de produções (GLANZEL, 2003).

A bibliometria constitui-se de um conjunto de leis que estudam quantitativamente o comportamento da informação registrada:

Assim, os estudos bibliométricos investigam o comportamento do conhecimento e da literatura, visando basicamente a análise quantitativa do conhecimento registrado, quer sejam: da produção científica dos autores, da produtividade de periódicos sobre determinado assunto, de mensuração e avaliações quantitativas dos processos referentes à utilização de documentos, análise de coautorias, rede de comunicações 
científicas, análise de avaliação da produção cientifica, cálculo do fator de impacto, etc... (ALVES, 2009, p. 106).

Indicadores bibliométricos são indicadores-produto (ou ainda indicadores de eficácia) quando se referem a resultados mais imediatos das políticas com a produção de artigos em ciência e tecnologia ou número de patentes (MUGNAINI; JANNUZZI; QUONIAM, 2004, p. 124). A bibliometria tem abrangência interdisciplinar ou multidisciplinar e pode ser aplicada a diversas áreas do conhecimento e possui como principal objetivo o desenvolvimento de indicadores cada vez mais confiáveis.

\section{PRODUÇÃo CIENTíFICA E PRODUTIVIDADE CIENTíFICA}

Botomé (1996, p. 120), ao tratar das implicações da pesquisa científica no que se refere às relações entre produção científica e as formas de acesso ao que foi produzido, argumenta que tornar o conhecimento produzido acessível é uma etapa do próprio processo de pesquisa e não outra coisa, algo alheio e diferente de pesquisar. Produzir algo só tem sentido se há um destino para o que é produzido. O que faz com que a pesquisa tenha uma razão de ser é a sua efetiva contribuição para melhorar as relações das pessoas com sua realidade, as situações com que se defrontam, elevando a qualidade de suas vidas.

Meadows (1999) destacou a importância da produtividade científica para a consolidação de novos conhecimentos e das diferentes áreas do saber. $\mathrm{Na}$ mesma perspectiva, relacionou produção científica à qualidade e a quantidade do que é produzido. Para tanto, há de se compreender os seus fundamentos, tal qual Carlotto e Câmara (2008), quando afirmam que a compreensão dos processos de produção científica de uma determinada área do saber permite a organização de um montante de informações disponíveis, integrando diferentes perspectivas. Em uma relação semelhante, e ainda as especifidades das áreas do saber, Bufrem et al. (2007) justificaram que o fenômeno da produção científica parte da necessidade informacional em domínios específicos para promoção do ciclo de produção, organização e compartilhamentos de novos conhecimentos, que "contribuam para a compreensão da história e dos saberes acumulados da ciência que se está analisando" (BUFREM et al., 2007, p. 42). Ainda sobre os 
benefícios dos estudos relacionados à produção científica, bem como seus impactos para a comunidade científica como um todo, Bretani et al. (2011), enfocavam que seus resultados impulsionam a ciência, tecnologia e competitividade, contribuindo para políticas de investimento nestes setores e para a análise da dinâmica das áreas científicas, bem como para a compreensão de áreas emergentes ou consolidadas.

A produção científica é divulgada em diferentes canais de comunicação, cuja preferência varia em função das características e natureza das áreas do saber, podendo ser em eventos, periódicos científicos, resumos, anais, entre outros (MOREIRA, 2017). A produção científica divulgada em periódicos científicos é importante para a constituição da ciência no âmbito acadêmico ao tornar o artigo um poderoso veículo de disseminação da informação científica (SILVEIRA, 2012). A publicação torna-se, portanto, processo elementar das atividades de comunicação e produção científica e cultural, como força motriz, à medida que é recuperada e compartilhada, retroalimentando em ciclo de produção de novos conhecimentos. Por outro lado, como afirmaram Mugnaini, Carvalho e Campanatti-Ortiz (2006), a produção científica não se restringe aos periódicos científicos como canais de comunicação, reúne todas as atividades de pesquisa, ensino e prática, resultando em serviços, métodos, técnicas e tecnologias em ações que se estendem à comunidade como um todo. Isso quer dizer que, tal qual Witter (1989), mesmo que a produção científica se concretize predominantemente por meio dos canais de comunicação em livros, artigos, teses, dissertações e revistas especializadas, há espaços significativos para divulgação científica em resumos em anais de congressos e eventos similares. Ou seja,

a produção científica está relacionada com a atuação dos cursos de pós-graduação, quer pelo seu fazer científico, quer pelo seu papel na formação de professores e pesquisadores que irão atuar em outras entidades, universitárias ou não. Seu produto é relevante, inclusive como veículo para a mudança da dependência para a independência científica e tecnológica e, consequentemente, econômica e política (WITTER, 1989, p. 29).

Se a análise sobre produtividade científica estiver com enfoque na América Latina, segundo Schmitt, Bertoldi e Mazo (2017), o Brasil é um dos países com maior nível de produtividade científica, cuja principal fonte de 
produção está nas atividades dos grupos de pesquisa. Se considerado o contexto dos grupos formais de pesquisa, ou seja, aqueles cadastrados no Diretório de Grupos de Pesquisa do Conselho Nacional de Desenvolvimento Científico e Tecnológico, cabe a inferência de que tal destaque seja resultado da organização de PPG. Isso porque, as universidades se estruturam como espaços de efetivas atividades de pesquisa e desenvolvimento e, segundo Silva, Casimiro e Duarte (2016, p. 15), os seus grupos de pesquisa são "responsáveis pela investigação de temáticas relevantes no âmbito científico, conduzem o debate e acirram o saber-fazer, contribuindo, sobremaneira, para a construção de conhecimentos."

Witter (2001) factuou a produção científica como de longa história, mas esparsa e com significativas interrupções, com esboço mais sistemático na década de 60 , cuja preocupação com a investigação científica tem sido incrementada desde a década de 90. Isso demonstra a construção de indicadores de maturidade científica das diferentes áreas (OLIVEIRA, 2004), visto que delimita todas as atividades de pesquisa, as quais sejam busca, uso, criação e produção de conhecimento em processos colaborativos, participativos, dinâmicos, permeado de relações e alimentado pelas publicações científicas (SILVA; PINHEIRO, 2008).

A produção científica possibilita o compartilhamento de novos conhecimentos resultantes das pesquisas realizadas, proporcionando interação, visibilidade, credibilidade, reconhecimento e prestígio nas comunidades científicas (MOREIRA; VILAN FILHO; MUELLER, 2015). Ainda nesta perspectiva factual, Vercesi (2002) afirma que dentre os fatores que influenciaram a produção científica brasileira está o avanço da consolidação das políticas de pós-graduação implantadas nas universidades do país. Ainda assim, a produtividade científica nos PPG segue a Lei de Lotka, com premissa elementar de que "alguns pesquisadores publicam muito e muitos publicam pouco" (VOOS, 1974), por meio de estabelecimento de redes e índices de produção científica (ALVARADO, 2009). 


\section{PPG E RELAÇÕES SOCIAIS DE PRODUÇÃO CIENTÍFICA}

Segundo Horta e Moraes (2005), os cursos stricto sensu deixaram de ser objetos isolados de avaliação para serem compreendidos como PPG, com mobilidade internacional, excelência acadêmico-científica, organicidade entre linhas de pesquisa, projetos, estrutura curricular, publicações, teses e dissertações, priorizando espaços para produção científica e formação de pesquisadores. Tais pesquisadores estruturam redes de participação e colaboração que resultam em processos de coautoria, concentrando relações em torno de linhas de pesquisa para as atividades de produção do conhecimento científico (ROSSONI; GUARIDO FILHO, 2009).

Segundo Dantas (2004), voltando à questão da avaliação dos PPG no Brasil, se estabelecem integrações entre agências de fomento à pesquisa científica, definindo políticas de ciência e tecnologia em várias áreas do saber e reavaliando os critérios avaliativos dos programas, valorizando indicadores de medida do impacto social do conhecimento produzido nos cursos de pósgraduação. $O$ envolvimento entre atores envolvidos nos processos de produção de conhecimento, além da "elaboração integrada de planos nacionais de pósgraduação e conferências nacionais de ciência, tecnologia e inovação, pode contribuir para um melhor direcionamento social das ações científicas adequadas à realidade brasileira" (DANTAS, 2004).

Evidências do crescimento de cooperação entre pesquisadores e programas de pós-graduação no âmbito da produção científica têm favorecido a compreensão da construção do conhecimento científico não como empreendimento individual, mas imerso em redes de relacionamentos (ROSSONI; GUARIDO FILHO, 2009, p. 367).

A partir desta imersão em redes de relacionamentos, a responsabilidade social dos PPG requer uma avaliação sistemática de seu papel no âmbito da produção científica e no contexto das instituições de educação superior, públicas ou privadas, estabelecendo intercessões entre ensino, pesquisa, extensão e gestão. As atividades científicas em pesquisa e desenvolvimento permite a formação de pesquisadores stricto sensu com potencial para produção de conhecimento com impacto nacional e internacional, subsidiada por PPG. Tal 
como afirmaram Botomé e Kubo (2002), os PPG precisam desenvolver habilidades e competências que permitam a transformação do conhecimento científico mais recente e de boa qualidade em atuações profissionais e aplicadas significativas para a sociedade como um todo (BOTOMÉ; KUBO, 2002).

A correlação social da produção científica permite a promoção de atividades e conceitos interdisciplinares decorrentes da participação e da colaboração entre os pesquisadores, que, segundo Silva et al. (2006), proporcionam análise e estruturação de campos de conhecimentos. Para Burt (1992), tal mediação e intercâmbio de informações entre os participantes no processo de produção científica são importantes para que se entendam os relacionamentos interagências, assim como afirmou Maciel (2007), sobre a prevalência de interesses e interdependência dos pesquisadores nas estruturas de colaboração, neste caso, aplicáveis aos PPG.

\section{METODOLOGIA}

Este estudo tem abordagem metodológica quantitativa nos processos investigativos que pressupõem a recuperação de dados mensuráveis e objetivos (PAYNE; PAYNE, 2004). Para Creswell (2010, p. 26) a "pesquisa quantitativa é um meio para testar teorias objetivas, examinando a relação entre as variáveis". Por outro lado, esta pesquisa também tem abordagem qualitativa nos processos que envolvem investigação acerca das impressões dos participantes sobre as variáveis que constituem este projeto de pesquisa.

Para os aspectos quantitativos, foi adotada a bibliometria que, segundo Araújo (2006, p. 12), é uma "técnica quantitativa e estatística de medição dos índices de produção e disseminação do conhecimento científico". Sobre os elementos fundamentais da pesquisa, este estudo apoia-se na taxonomia de Babbie (2009).

As fontes de informações que foram adotadas para este estudo são o cadastro dos PPG brasileiros, o Diretório de Grupos de Pesquisa do CNPq e a Plataforma Lattes, também do CNPq. Também foi utilizado o ScriptLattes para acesso e compilação das produções científicas do conjunto de pesquisadores e estudantes definido para este estudo. 


\section{PRODUÇÃO CIENTÍFICA DOS MEMBROS DOS PPGS NAS AIBR}

O Quadro 1 apresenta a distribuição das frequências da produção científica total por PPG AIBR, conforme proposto pelo primeiro objetivo específico deste estudo. Observa-se que, dos 20 PPG AIBR, a Universidade Federal de São Carlos figura a primeira posição, com frequência absoluta de 10.660 produções científicas dentro do período de estudo (1992 - 2017), representando $12,73 \%$ de toda a produção científica dos PPG estudados. Destaca-se que o PPG da Universidade Federal de São Carlos é relativamente novo, quando comparado aos demais PPG de Ciência da Informação no país. Ainda assim, apresenta a maior frequência de produção científica, considerando as AIBR. Os demais Quadros, que apresentam a quantidade de membros (considerando estudantes), podem ajudar a explicar este fenômeno.

A Universidade de São Paulo e a Universidade Estadual Paulista ocupam a segunda e a terceira posições no quadro de distribuição do quantitativo de produção científica por PPG. Neste caso, a Universidade de São Paulo possui, considerando o período estudado, 8.578 produções científicas. Já a Universidade Estadual Paulista possui 8.381 produções científicas, considerando o mesmo período (1992-2017) e os mesmos tipos de participantes (pesquisadores e estudantes). Isso representa, respectivamente, 10,24\% e $10,01 \%$ do quadro de distribuição do quantitativo de produção científica por PPG nas AIBR. Destaca-se, também, que mais de $50 \%$ da produção científica é originária de apenas 5 PPG, sendo Universidade Federal de São Carlos, Universidade de São Paulo, Universidade Estadual Paulista, Universidade Federal do Estado do Rio de Janeiro e Universidade de Brasília (Quadro 1).

Quadro 1 - Distribuição da produção científica total por PPG AIBR 


\begin{tabular}{|c|c|c|c|c|c|}
\hline \multirow[b]{2}{*}{ SEQ } & \multirow[b]{2}{*}{ IES } & \multicolumn{2}{|c|}{ Frequências Simples } & \multicolumn{2}{|c|}{ Frequências Acumuladas } \\
\hline & & $\begin{array}{l}\text { PRODUÇÃO } \\
\text { CIENTÍFICA }\end{array}$ & $\begin{array}{c}\text { PRODUÇÃO } \\
\text { Relativa (\%) }\end{array}$ & $\begin{array}{c}\text { PRODUAÇÃO } \\
\text { CIENTÍFICA } \\
\text { Acumulada }\end{array}$ & $\begin{array}{c}\text { Frequência } \\
\text { Relativa } \\
\text { Acumulada } \\
(\%) \\
\end{array}$ \\
\hline 1 & UFSCAR & 10660 & 12,73 & 10660 & 12,73 \\
\hline 2 & USP & 8578 & 10,24 & 19238 & 22,98 \\
\hline 3 & UNESP & 8381 & 10,01 & 27619 & 32,98 \\
\hline 4 & UNIRIO & 7995 & 9,55 & 35614 & 42,53 \\
\hline 5 & UNB & 7708 & 9,21 & 43322 & 51,74 \\
\hline 6 & UFPB & 6779 & 8,10 & 50101 & 59,83 \\
\hline 7 & UFSC & 5544 & 6,62 & 55645 & 66,46 \\
\hline 8 & UFMG & 4841 & 5,78 & 60486 & 72,24 \\
\hline 9 & UFBA & 4559 & 5,44 & 65045 & 77,68 \\
\hline 10 & UFPE & 4144 & 4,95 & 69189 & 82,63 \\
\hline 11 & UFF & 3520 & 4,20 & 72709 & 86,83 \\
\hline 12 & UFC & 2639 & 3,15 & 75348 & 89,99 \\
\hline 13 & UEL & 1991 & 2,38 & 77339 & 92,36 \\
\hline 14 & FUFSE & 1753 & 2,09 & 79092 & 94,46 \\
\hline 15 & UFRJ & 1067 & 1,27 & 80159 & 95,73 \\
\hline 16 & UFPA & 994 & 1,19 & 81153 & 96,92 \\
\hline 17 & UDESC & 865 & 1,03 & 82018 & 97,95 \\
\hline 18 & UFRN & 830 & 0,99 & 82848 & 98,94 \\
\hline 19 & UFCA & 610 & 0,73 & 83458 & 99,67 \\
\hline 20 & FCRB & 275 & 0,33 & 83733 & 100,00 \\
\hline & TOTAL & 83733 & 100,00 & & \\
\hline
\end{tabular}

Fonte: Dados da pesquisa.

Onde: IES = Instituição de Educação Superior; PRODUÇÃO CIENTÍFICA = Total da produção científica por IES que tem PPG AIBR, considerando a produção de pesquisadores e estudantes no período de 1992-2017, admitindo dupla contagem; PRODUÇÃO Relativa (\%) = Percentual de de produção científica por IES, considerando o TOTAL de produção científica.

Ressalta-se o PPG com maior quantidade de produção científica, neste caso, a Universidade Federal de São Carlos e o programa com a menor produção científica dentre os estudados, com 275 (Fundação Casa de Rui Barbosa), considerando o mesmo período de análise (1992 - 2017). Considerando o total de produção científica dos 20 PPG juntos $(n=83733)$, a média de produção científica é de 4186,65 . Outros dados podem ser explorados com relação ao Quadro 1, no que pertence à produção científica dos PPG AIBR, por IES, pelo tempo de formação dos respectivos programas. PPG AIBR mais jovens, ou seja, com ano de formação inferior a 5 anos (tendo como base $o$ ano de 2018), apresentaram maior produção científica do que PPG AIBR mais tradicionais, aqueles mais antigos.

A produção científica dos PPG AIBR da Universidade Federal de São Carlos (ano de formação 2016) e da Universidade Estadual Paulista (ano de 
formação 2013), por exemplo, apresentaram produção científica maior que os PPG AIBR da Universidade de Brasília e da Universidade Federal de Minas Gerais, com anos de formação, respectivamente, em 1978 e 1997. Este dado é interessante, principalmente, quando consideramos que os participantes de PPG podem figurar em um ou muitos PPG, visto que podem colaborar em grupos de estudo, grupos de pesquisa ou outras organizações científicas formais. Da mesma forma, sua produção científica individual pode compor a produção científica de um ou muitos PPG.

\section{PRODUÇÃO CIENTÍFICA DOS MEMBROS DOS PPGS NAS AIBR POR CANAL DE COMUNICAÇÃO}

O Quadro 2 apresenta a distribuição das frequências da produção científica dos PPG AIBR por canal de comunicação. A partir desse quadro é possível fazer pelo menos dois tipos de análises específicas. Verticalmente, observa-se a quantidade de produção científica por canal de comunicação entre os PPG estudados. Horizontalmente, o Quadro 2 mostra a distribuição da produção científica por canal de comunicação e por PPG AIBR.

Praticamente todos os programas estudados divulgam sua produção científica majoritariamente em trabalhos completos em anais de congresso. $O$ PPG da Universidade Federal de Santa Catarina, por exemplo, tem quase 50\% de sua produção científica publicada em trabalhos completos em anais de congressos. A exceção seria a Universidade Federal do Rio de Janeiro, cuja primeira escolha para divulgação científica são artigos de periódicos, com $31,87 \%$. Ainda assim, o canal de comunicação de que está na segunda posição para a Universidade Federal do Rio de Janeiro é trabalho completo em anais de congresso.

Por meio da análise vertical, ou seja, a quantidade de produção científica por canal de comunicação entre os PPG estudados, percebe-se que a produção científica mais presente em artigos completo de periódico é a da Universidade Federal de São Carlos, com 2.412 produções neste canal de comunicação. Por outro lado, considerando a produção científica publicado livro, com análise entre os PPG, a Universidade Federal do Estado do Rio de Janeiro é a que mais 
procura este canal de comunicação, com 603 publicações. Entrando, isto não é verdade se considerarmos apenas o PPG em Ciência da Informação da Universidade Federal do Estado do Rio de Janeiro, como veremos mais adiante. Ainda sob análise vertical do Quadro 2, tratando agora da produção científica publicada em Capítulos de Livros, a Universidade Estadual Paulista foi a IES cujo PPG AIBR foi o que mais publicou neste canal de comunicação, com 1.159 publicações, considerando o período de 1992 - 2017 e a produção total dos seus pesquisadores e estudantes. O PPG em Ciência da Informação da Universidade Estadual Paulista não data de 1992, mas há de se considerar que os pesquisadores e estudantes que compõem ou compuseram o referido PPG têm sua produção científica pregressa contada para fins deste estudo. Com os mesmos parâmetros de análise, a Universidade Estadual Paulista é a IES que tem o PPG AlBR que mais publicou em Resumos Expandidos, com 539 produções científicas neste mesmo canal de comunicação.

Partindo para uma análise horizontal do Quadro 2, os PPG AIBR, independente da produtividade científica, considerando os parâmetros deste estudo, têm maior proporção de publicação em Trabalhos Completos de Anais de Congressos. A frequência média de produção científica neste canal de comunicação é de cerca de $35 \%$. Por outro lado, por IES, os canais de comunicação Livros e Resumos Expandidos, foram os que menos publicaram a produção científica dos PPG AIBR, por IES, com frequência média de cerca de $5 \%$ para ambos os canais de comunicação.

\section{Quadro 2 - Distribuição da produção científica dos PPG AIBR por canal de comunicação}

\begin{tabular}{|c|c|c|c|c|c|c|c|c|c|c|c|c|c|}
\hline IES & $\begin{array}{c}\text { canal } \\
\text { AP }\end{array}$ & $\begin{array}{l}\text { FR } \\
(\%)\end{array}$ & $\begin{array}{l}\text { cana } \\
\text { l LV }\end{array}$ & $\begin{array}{l}\text { FR } \\
(\%)\end{array}$ & $\begin{array}{c}\text { canal } \\
\text { CL }\end{array}$ & $\begin{array}{c}\text { FR } \\
(\%)\end{array}$ & $\begin{array}{l}\text { canal } \\
\text { TCA }\end{array}$ & $\begin{array}{c}\text { FR } \\
(\%)\end{array}$ & $\begin{array}{l}\text { canal } \\
\text { REA }\end{array}$ & $\begin{array}{l}\text { FR } \\
(\%)\end{array}$ & $\begin{array}{l}\text { canal } \\
\text { RPA }\end{array}$ & $\begin{array}{c}\text { FR } \\
(\%)\end{array}$ & Total \\
\hline UFSCAR & 2412 & $\begin{array}{r}22,6 \\
3 \\
\end{array}$ & 516 & 4,84 & 1159 & 10,87 & 3568 & 33,47 & 426 & 4,00 & 2579 & 24,19 & 10660 \\
\hline USP & 2332 & $\begin{array}{r}27,1 \\
9 \\
\end{array}$ & 481 & 5,61 & 1264 & 14,74 & 2761 & 32,19 & 307 & 3,58 & 1433 & 16,71 & 8578 \\
\hline UNESP & 1885 & $\begin{array}{r}22,4 \\
9 \\
\end{array}$ & 317 & 3,78 & 1279 & 15,26 & 2569 & 30,65 & 539 & 6,43 & 1792 & 21,38 & 8381 \\
\hline UNIRIO & 2183 & $\begin{array}{r}27,3 \\
0 \\
\end{array}$ & 603 & 7,54 & 184 & 14,81 & 2478 & 30,99 & 417 & 5,22 & 1130 & 14,13 & 7995 \\
\hline UNB & 2390 & $\begin{array}{r}31,0 \\
1 \\
\end{array}$ & 593 & 7,69 & 1024 & 13,28 & 2610 & 33,86 & 245 & 3,18 & 846 & 10,98 & 7708 \\
\hline UFPB & 2087 & $\begin{array}{r}30,7 \\
9 \\
\end{array}$ & 277 & 4,09 & 774 & 11,42 & 2468 & 36,41 & 272 & 4,01 & 901 & 13,29 & 6779 \\
\hline
\end{tabular}




\begin{tabular}{|c|c|c|c|c|c|c|c|c|c|c|c|c|c|}
\hline UFSC & 1548 & $\begin{array}{r}27,9 \\
2\end{array}$ & 234 & 4,22 & 542 & 9,78 & 2487 & 44,86 & 141 & 2,54 & 592 & 10,68 & 5544 \\
\hline UFMG & 1620 & $\begin{array}{r}33,4 \\
6 \\
\end{array}$ & 178 & 3,68 & 456 & 9,42 & 1924 & 39,74 & 210 & 4,34 & 453 & 9,36 & 4841 \\
\hline UFBA & 1018 & $\begin{array}{r}22,3 \\
3 \\
\end{array}$ & 289 & 6,34 & 655 & 14,37 & 1712 & 37,55 & 150 & 3,29 & 735 & 16,12 & 4559 \\
\hline UFPE & 1014 & $\begin{array}{r}24,4 \\
7 \\
\end{array}$ & 219 & 5,28 & 492 & 11,87 & 1658 & 40,01 & 232 & 5,60 & 529 & 12,77 & 4144 \\
\hline UFF & 979 & $\begin{array}{r}27,8 \\
1 \\
\end{array}$ & 237 & 6,73 & 544 & 15,45 & 1051 & 29,86 & 122 & 3,47 & 587 & 16,68 & 3520 \\
\hline UFC & 644 & $\begin{array}{r}24,4 \\
0 \\
\end{array}$ & 80 & 3,03 & 305 & 11,56 & 743 & 28,15 & 236 & 8,94 & 631 & 23,91 & 2639 \\
\hline UEL & 526 & $\begin{array}{r}26,4 \\
2 \\
\end{array}$ & 53 & 2,66 & 252 & 12,66 & 704 & 35,36 & 137 & 6,88 & 319 & 16,02 & 1991 \\
\hline FUFSE & 387 & $\begin{array}{r}22,0 \\
8\end{array}$ & 98 & 5,59 & 162 & 9,24 & 744 & 42,44 & 49 & 2,80 & 313 & 17,86 & 1753 \\
\hline UFRJ & 340 & $\begin{array}{r}31,8 \\
7\end{array}$ & 68 & 6,37 & 133 & 12,46 & 261 & 24,46 & 40 & 3,75 & 225 & 21,09 & 1067 \\
\hline UFPA & 208 & $\begin{array}{r}20,9 \\
3 \\
\end{array}$ & 60 & 6,04 & 173 & 17,40 & 272 & 27,36 & 62 & 6,24 & 219 & 22,03 & 994 \\
\hline UDESC & 299 & $\begin{array}{r}34,5 \\
7 \\
\end{array}$ & 25 & 2,89 & 46 & 5,32 & 299 & 34,57 & 15 & 1,73 & 181 & 20,92 & 865 \\
\hline UFRN & 164 & $\begin{array}{r}19,7 \\
6 \\
\end{array}$ & 30 & 3,61 & 88 & 10,60 & 359 & 43,25 & 42 & 5,06 & 147 & 17,71 & 830 \\
\hline UFCA & 137 & $\begin{array}{r}22,4 \\
6 \\
\end{array}$ & 11 & 1,80 & 40 & 6,56 & 223 & 36,56 & 95 & 15,57 & 104 & 17,05 & 610 \\
\hline FCRB & 65 & $\begin{array}{r}23,6 \\
4 \\
\end{array}$ & 40 & 14,55 & 62 & 22,55 & 79 & 28,73 & 6 & 2,18 & 23 & 8,36 & 275 \\
\hline TOTAL & $\begin{array}{r}2223 \\
8 \\
\end{array}$ & & 4409 & & 10634 & & 28970 & & 3743 & & 13739 & & 83733 \\
\hline
\end{tabular}

Fonte: Dados da pesquisa.

Onde: $\mathrm{AP}=$ artigos completos em periódicos; $\mathrm{LV}=$ livros; $\mathrm{CL}=$ capítulo de livros; $\mathrm{TCA}=$ trabalhos completos em anais de congresso; REA = resumos expandidos; RPA = resumos publicados em anais; $\mathrm{FR}(\%)=$ Frequência relativa. As cores presentes no Quadro representam um método de classificação condicional por escala de cor. Quanto mais verde, maior a ocorrência; quanto mais vermelha, menor a ocorrência.

\section{PRODUÇÃO CIENTÍFICA DOS MEMBROS DOS PPGS NAS AIBR POR QUANTIDADE DE MEMBROS}

O Quadro 3 mostra que não há uma relação específica entre volume de pesquisadores e estudantes (membros) e o volume da produção científica. Ou seja, não necessariamente um PPG que tenha maior número de membros será o mais produtivo. O PPG estudado da Universidade Federal do Estado do Rio de Janeiro, por exemplo, é o que tem a maior quantidade de membros (366) e, entretanto, figura na quarta posição dos programas mais produtivos, proporcionalmente. Da mesma forma, a Universidade de Brasília que tem o segundo maior PPG em Ciência da Informação em número de membros 
(pesquisadores/estudantes) ocupa apenas a sétima colocação entre os programas mais produtivos, com 26,72 produção científica por participante. Por outro lado, o PPG de Ciência da Informação da Universidade Estadual Paulista não é o que detém o maior quantitativo de participantes (239), mas é, proporcionalmente, o mais produtivo, com a relação de 33,35 produção científica por participante.

Considerando todos os 20 PPG AIBR estudados, a média de produção científica por quantidade de participantes, os quais sejam pesquisadores e estudantes, é 24,11. O programa que tem a maior relação de produção científica por quantidade de membros é a Universidade Estadual Paulista, com 33,45 por participante (pesquisador/estudante). O programa que tem a menor relação de produção científica por quantidade de membros é o da Fundação Casa de Rui Barbosa, com 16,17 produção científica por membro (pesquisador/estudante).

\section{Quadro 3 - Proporção de produção científica dos PPG AIBR por quantidade de membros}

\begin{tabular}{r|l|r|r|r|r}
\hline $\begin{array}{c}\text { SE } \\
\mathbf{Q}\end{array}$ & \multicolumn{1}{|c|}{ IES } & $\begin{array}{c}\text { QTD } \\
\text { MEMBROS }\end{array}$ & $\begin{array}{c}\text { Produção Relativa } \\
\mathbf{( \% )}\end{array}$ & \multicolumn{1}{c|}{$\begin{array}{c}\text { Produção } \\
\text { Total }\end{array}$} & $\begin{array}{c}\text { Produção } \\
\text { Científica } \\
\text { Qtd Membros }\end{array}$ \\
\hline 1 & UNESP & 239 & 7,60 & 8381 & 33,45 \\
\hline 2 & UFBA & 254 & 8,07 & 4559 & 33,00 \\
\hline & UFSCA & 238 & 7,57 & 10660 & 32,39 \\
3 & R & 366 & 11,63 & 7995 & 29,13 \\
\hline 4 & UNIRIO & 233 & 7,41 & 6779 & 29,09 \\
\hline 5 & UFPB & 206 & 6,55 & 4144 & 26,91 \\
\hline 6 & UFPE & 321 & 10,20 & 7708 & 26,72 \\
\hline 7 & UNB & 140 & 4,45 & 3520 & 25,14 \\
\hline 8 & UFF & 200 & 6,36 & 4841 & 24,21 \\
\hline 9 & UFMG & 198 & 6,29 & 5544 & 23,03 \\
\hline 10 & UFSC & 27 & 0,86 & 830 & 22,59 \\
\hline 11 & UFRN & 117 & 3,72 & 1991 & 22,56 \\
\hline 12 & UEL & 195 & 6,20 & 8578 & 21,25 \\
\hline 13 & USP & 40 & 1,27 & 610 & 20,75 \\
\hline 14 & UFCA & 49 & 1,56 & 865 & 20,29 \\
\hline 15 & UDESC & 53 & 1,68 & 1067 & 20,13 \\
\hline 16 & UFRJ & 44 & 1,40 & 994 & 19,66 \\
\hline 17 & UFPA & 109 & 3,46 & 2639 & 18,27 \\
\hline 18 & UFC & 100 & 3,18 & 1753 & 17,53 \\
\hline 19 & FUFSE & 17 & 0,54 & 275 & 16,18 \\
\hline 20 & FCRB & $\mathbf{3 1 4 6}$ & $\mathbf{1 0 0 , 0 0}$ & $\mathbf{8 3 7 3 3}$ & $\mathbf{2 6 , 6 2}$ \\
\hline \multicolumn{1}{|l}{ TOTAL } & & & \\
\hline
\end{tabular}

Fonte: Dados da pesquisa.

As cores presentes no Quadro representam um método de classificação condicional por escala de cor. Quanto mais verde, maior a ocorrência; quanto mais vermelha, menor a ocorrência. Os 
nomes completos das IES estão descritos no Apêndice I. Qtd Membros $=(\Sigma$ Qtd pesquiadores e estudantes) por PPG AIBR.

Em se tratando da relação entre a produção científica dos PPG AIBR por quantidade de membros, assim como apresentado no Quadro 4, percebe-se que, se a frequência relativa for apenas pela quantidade de membros e não pela proporção, da mesma forma, os programas mais populosos não são os mais produtivos.

\section{Quadro 4 - Relação entre produção científica dos PPG AIBR por quantidade de membros}

\begin{tabular}{l|r|r|r|l|l}
\hline \multicolumn{1}{c|}{ IES } & $\begin{array}{c}\text { QTD } \\
\text { MEMBROS }\end{array}$ & $\begin{array}{c}\text { Produção Relativa } \\
(\%)\end{array}$ & $\begin{array}{c}\text { Produção } \\
\text { Total }\end{array}$ & \multicolumn{1}{c}{ IES } & $\begin{array}{r}\text { PRODUÇ̃̃ } \\
\text { CIENTÍFICA }\end{array}$ \\
\hline UNIRIO & 366 & 11,63 & 7995 & UFSCAR & 8578 \\
\hline UNB & 321 & 10,20 & 7708 & USP & 8381 \\
\hline UFBA & 254 & 8,07 & 4559 & UNESP & 7995 \\
\hline UNESP & 239 & 7,60 & 8381 & UNIRIO & 7708 \\
\hline UFSCAR & 238 & 7,57 & 10660 & UNB & 6779 \\
\hline UFPB & 233 & 7,41 & 6779 & UFPB & 5544 \\
\hline UFPE & 206 & 6,55 & 4144 & UFSC & 4841 \\
\hline UFMG & 200 & 6,36 & 4841 & UFMG & 4559 \\
\hline UFSC & 198 & 6,29 & 5544 & UFBA & 4144 \\
\hline USP & 195 & 6,20 & 8578 & UFPE & 3520 \\
\hline UFF & 140 & 4,45 & 3520 & UFF & 2639 \\
\hline UEL & 117 & 3,72 & 1991 & UFC & 1991 \\
\hline UFC & 109 & 3,46 & 2639 & UEL & 1753 \\
\hline FUFSE & 100 & 3,18 & 1753 & FUFSE & 1067 \\
\hline UFRJ & 53 & 1,68 & 1067 & UFRJ & 994 \\
\hline UDESC & 49 & 865 & UFPA & 865 \\
\hline UFPA & 44 & 994 & UDESC & 830 \\
\hline UFCA & 40 & 610 & UFRN & 610 \\
\hline UFRN & 27 & 830 & UFCA & 275 \\
\hline FCRB & 17 & 275 & FCRB & \\
\hline TOTAL & $\mathbf{3 1 4 6}$ & 1,40 & $\mathbf{8 3 7 3 3}$ & & \\
\hline
\end{tabular}

Fonte: Dados da pesquisa.

As cores presentes no Quadro representam um método de classificação condicional por escala de cor. Quanto mais verde, maior a ocorrência; quanto mais vermelha, menor a ocorrência. Qtd Membros $=(\Sigma$ Qtd pesquiadores e estudantes $)$ por PPG AIBR.

A Universidade Federal de São Carlos, por exemplo, tem um PPG com 238 membros - quinta colocação entre os mais populosos - e é o programa com maior produção científica. Por outro lado, o PPG da Universidade Federal da Bahia, que em número de membros ocupa a terceira posição entre os 20 PPG AIBR estudados, cai para situação mediana em números de produção científica.

Um dado interessante é que a Universidade de São Paulo, que está na décima posição em número de membros, tem o PPG na segunda posição em 
quantidade de produção científica. Se considerados os 20 PPG estudados, há uma média de 157,3 participantes por programa. O PPG de Ciência da Informação da UNIRIO é o que contém maior quantidade de membros, sendo 366 membros entre pesquisadores e estudantes. Por outro lado, a Fundação Casa de Rui Barbosa é a que reúne menor número de membros no programa, sendo 17 membros.

O Quadro 5 apresenta a relação entre o tipo de participante e a produção científica dos PPG AIBR. Considera-se como tipo de participante: pesquisadores e estudantes, perfis do Diretório de Grupos de Pesquisa do CNPq. Para este estudo não foram considerados os técnicos.

\section{Quadro 5 - Relação entre tipo de participante e a produção científica dos PPG AIBR}

\begin{tabular}{|c|c|c|c|c|}
\hline IES & Qtd P & Qtd E & QTD MEMBROS & Produção Total \\
\hline UNIRIO & 169 & 197 & 366 & 7995 \\
\hline UNB & 189 & 132 & 321 & 7708 \\
\hline UFBA & 115 & 139 & 254 & 4559 \\
\hline UNESP & 116 & 123 & 239 & 8381 \\
\hline UFSCAR & 130 & 108 & 238 & 10660 \\
\hline UFPB & 114 & 119 & 233 & 6779 \\
\hline UFPE & 99 & 107 & 206 & 4144 \\
\hline UFMG & 127 & 73 & 200 & 4841 \\
\hline UFSC & 79 & 119 & 198 & 5544 \\
\hline USP & 117 & 78 & 195 & 8578 \\
\hline UFF & 82 & 58 & 140 & 3520 \\
\hline UEL & 50 & 67 & 117 & 1991 \\
\hline UFC & 70 & 39 & 109 & 2639 \\
\hline FUFSE & 53 & 47 & 100 & 1753 \\
\hline UFRJ & 22 & 31 & 53 & 1067 \\
\hline UDESC & 24 & 25 & 49 & 865 \\
\hline UFPA & 37 & 7 & 44 & 994 \\
\hline UFCA & 19 & 21 & 40 & 610 \\
\hline UFRN & 20 & 7 & 27 & 830 \\
\hline FCRB & 16 & 1 & 17 & 275 \\
\hline MÉDIA & 82,4 & 74,9 & 157,3 & 26,62 \\
\hline TOTAL & 1648 & 1498 & 3146 & 83733 \\
\hline
\end{tabular}

Fonte: Dados da pesquisa.

As cores presentes no Quadro representam um método de classificação condicional por escala de cor. Quanto mais verde, maior a ocorrência; quanto mais vermelha, menor a ocorrência. Qtd Membros $=(\Sigma$ Qtd pesquiadores e estudantes $)$ por PPG AIBR.

A média de pesquisadores por programa é de 82,4. O programa que possui maior número de pesquisadores é o da Universidade de Brasília, com 189 pesquisadores. O programa que possui menor número de pesquisadores é a 
Fundação Casa de Rui Barbosa, com 16 pesquisadores. Por outro lado, a média de estudantes por programa é de 74,9 estudantes. O programa que possui maior quantidade de estudantes, dentre os programas estudados, é da Universidade Federal do Estado do Rio de Janeiro, com 197 estudantes; e o programa com menor quantidade de estudantes é o da Fundação Casa de Rui Barbosa, com apenas 1 estudante. Cabe destacar a Universidade Federal de Santa Catarina que possui quantidade de estudantes superior à quantidade de pesquisadores, sendo 119 e 79 respectivamente, com produção científica total mediana (5544), porém bem à frente de mais de $50 \%$ dos programas estudados.

\section{PRODUÇÃO CIENTÍFICA DOS MEMBROS DOS PPG AIBR E O CONCEITO CAPES}

Do total de programas estudados, apenas 1 têm conceito CAPES 6 (Universidade Estadual Paulista); 3 programas têm conceito CAPES 5; 7 programas têm conceito CAPES 4; e 9 programas têm conceito CAPES 3. Cabe destacar que os conceitos aqui desenvolvidos são referentes à avaliação quadrienal CAPES até 2017.

Percebe-se, conforme o Quadro 6, em análise absoluta, que o programa com o maior conceito CAPES (6) - Universidade Estadual Paulista - é um dos mais produtivos. Nesta mesma perspectiva de análise, há programas mais produtivos que estão entre os que têm conceito CAPES 4. Entretanto, há de se considerar a proporcionalidade, visto que a quantidade de programas por conceito é diferente. Cabe destacar, também, que do rol de PPG AIBR estudados, o que apresenta maior produtividade não tem, para a quadrienal CAPES estudada, o maior conceito, sendo a UFSCAR, com conceito CAPES 3 e 10660 produções científicas de seus pesquisadores e estudantes, considerando o período de 1992-2017. 


\section{Quadro 6 - Relação entre a produção científica dos PPG AIBR e seus conceitos CAPES}

\begin{tabular}{c|l|r|c}
\hline \multicolumn{1}{c|}{ INSTITUIÇÃO } & PRODUÇÃO TOTAL & CONCEITO \\
\hline 1 & UNESP & 8381 & 6 \\
\hline \multicolumn{1}{|c|}{ Total C(6) } & 8381 & \\
\hline 2 & UFSC & 5544 & 5 \\
\hline 3 & UNB & 7708 & 5 \\
\hline 4 & UFMG & 4841 & 5 \\
\hline \multicolumn{1}{|c|}{ Total C(5) } & 18093 & \\
\hline 5 & UEL & 1991 & 4 \\
\hline 6 & UFRJ & 1067 & 4 \\
\hline 7 & UFBA & 4559 & 4 \\
\hline 8 & UFF & 3520 & 4 \\
\hline 9 & UFPB & 6779 & 4 \\
\hline 10 & UFPE & 4144 & 4 \\
\hline 11 & USP & 8578 & 4 \\
\hline \multicolumn{1}{|c|}{ Total C(4) } & 30638 & \\
\hline 12 & FCRB & 275 & 3 \\
\hline 13 & FUFSE & 1753 & 3 \\
\hline 14 & UDESC & 865 & 3 \\
\hline 15 & UFC & 2639 & 3 \\
\hline 16 & UFCA & 610 & 3 \\
\hline 17 & UFPA & 994 & 3 \\
\hline 18 & UFRN & 830 & 3 \\
\hline 19 & UFSCAR & 10660 & 3 \\
\hline 20 & UNIRIO & 7995 & 3 \\
\hline & Total C(3) & $\mathbf{2 6 6 2 1}$ & \\
\hline & & & \\
\hline
\end{tabular}

Fonte: Dados da pesquisa.

Onde: $\mathrm{C}=$ conceito. As cores presentes no Quadro representam um método de classificação condicional por escala de cor. Quanto mais verde, maior a ocorrência; quanto mais vermelha, menor a ocorrência.

Proporcionalmente, então, o programa mais produtivo é o que detém conceito CAPES 6 , sendo sua produção científica de 8.381 produções, compreendendo a Universidade Estadual Paulista. Com mesmo critério de análise, na segunda posição estão os programas com conceito CAPES 4, com produção científica média de 4928,5 por PPG AIBR.

Com base do quantitativo alto de produção científica, assim como apresentado no Quadro 6, cabe destacar, novamente que, para este estudo, considerou-se como produção científica dos PPG AIBR o somatório da produção científica dos participantes (pesquisadores e estudantes) dos PPG. Pressupõese que toda produção científica individual de um determinado participante esteja contida na produção científica do PPG ao qual ele participa. No caso do participante pertencer a mais de um PPG, admite-se, então, dupla contagem. Outro ponto relevante é que os dados apresentados se referem à base da 
Plataforma Lattes, conforme registros até 2017.

\section{PRODUÇÃO CIENTÍFICA DOS MEMBROS DOS PPG AIBR POR REGIÃO}

Considerando a produção científica dos membros dos PPG AIBR por distribuição geográfica, o Quadro 7 mostra que mais de $50 \%$ da produção está na Região Sudeste do país; um quarto da produção científica total está na Região Nordeste; o restante da produção científica está distribuído entre as demais Regiões do país.

Quadro 7 - Relação entre produção científica, PPG AIBR e UF

\begin{tabular}{c|l|r|c}
\hline Sequência & \multicolumn{1}{|c|}{ Instituição } & produção total & UF \\
\hline 1 & UFSCAR & 10660 & SP \\
\hline 2 & USP & 8578 & SP \\
\hline 3 & UNESP & 8381 & SP \\
\hline 4 & UNIRIO & 7995 & RJ \\
\hline 5 & UNB & 7708 & DF \\
\hline 6 & UFPB & 6779 & PB \\
\hline 7 & UFSC & 5544 & SC \\
\hline 8 & UFMG & 4841 & MG \\
\hline 9 & UFBA & 4559 & BA \\
\hline 10 & UFPE & 4144 & PE \\
\hline 11 & UFF & 3520 & RJ \\
\hline 12 & UFC & 2639 & CE \\
\hline 13 & UEL & 1991 & PR \\
\hline 14 & FUFSE & 1753 & SE \\
\hline 15 & UFRJ & 1067 & RJ \\
\hline 16 & UFPA & 994 & PA \\
\hline 17 & UDESC & 865 & SC \\
\hline 18 & UFRN & 830 & RN \\
\hline 19 & UFCA & 610 & CE \\
\hline 20 & FCRB & 275 & RJ \\
\hline & TOTAL & $\mathbf{8 3 7 3 3}$ & \\
\hline
\end{tabular}

Fonte: Dados da pesquisa.

Dentre os estados da Região Sudeste, São Paulo detém mais da metade da produção científica dos PPG AIBR.

\section{CONCLUSÕES}

O objetivo deste estudo foi descrever a produtividade científica dos PPG AIBR (Arquivologia, Biblioteconomia, Ciência da Informação, Documentação e Museologia), representada por seus membros pesquisadores e estudantes. Este 
objetivo foi alcançado e os dados revelaram um determinado padrão para a produção científica dos programas estudados.

A produção científica de poucos PPG AIBR representa mais de $50 \%$ da produção científica da área, tendo como programas mais produtivos: Universidade Federal de São Carlos, Universidade de São Paulo, Universidade Estadual Paulista, Universidade Federal do Estado do Rio de Janeiro e Universidade de Brasília, respectivamente.

Os PPG AIBR preferem divulgar sua produção científica em canais de comunicação de trabalhos completos em anais de congresso. Ainda no mesmo contexto, não há uma relação específica entre volume de pesquisadores e estudantes e o volume da produção científica. Ou seja, não necessariamente um PPG AIBR que tenha maior número de pesquisadores e estudantes será a mais produtiva. Percebe-se que, se a frequência relativa for apenas pela quantidade de membros e não pela proporção, da mesma forma, os programas mais populosos não são os mais produtivos.

Os PPG AIBR da Região Sudeste predominam como os mais produtivos, com mais de $50 \%$ da produção científica da área, seguidos dos programas da Região Nordeste, com $1 / 4$ da produção científica total dos PPG AIBR no país.

Por fim, os PPG AIBR mais qualificados pela CAPES, ou seja, com conceito 6 , ainda são os com maior produção científica, notando-se, também, que os programas com conceito CAPES 4 figuram a segunda posição entre os mais produtivos. De forma sumária, se pudéssemos definir um padrão para a produção científica dos PPG AIBR, com base nos dados deste estudo, seriam mais produtivos os PPG: (i) da Região Sudeste; (ii) mais qualificados, com conceito 6 da CAPES; e (iii) com preferência para divulgar sua produção científica em canais de comunicação de trabalhos completos em anais de congresso.

\section{REFERÊNCIAS}

ALMEIDA, E. C. E.; GUIMARÃES, J. A.; ALVES, I. T. G. Dez anos do Portal de Periódicos da CAPES: histórico, evolução e utilização. Revista Brasileira de Pós-Graduação, Brasília, v. 7, n. 13, p. 218-246, 2010. 
ALVARADO, R. U. A frente de pesquisa na literatura sobre a produtividade dos autores. Encontros Bibli: Revista Eletrônica de Biblioteconomia e Ciência da Informação, Florianópolis, v. 14, n. 28, p. 39-57, 2009.

ALVES, B. H. Abordagens métricas: análise da produção científica de artigos e rede de colaboração científica dos docentes do programa de pós-graduação em ciência da informação, na linha de pesquisa organização da informação da Unesp/Marília. Revista de Iniciação Científica da FFC, Marília, v. 9, n. 2, p. 104-115, 2009.

ARAÚJO, C. A. Bibliometria: evolução história e questões atuais. Em Questão, Porto Alegre, v. 12, n. 1, p. 11-32, jan./jun. 2006.

BABBIE, E. R. The Practice of Social Research. 12.ed. Wadsworth, 2009, p.436-440.

BOTOMÉ, S. P. Pesquisa alienada e Ensino alienante: o equívoco da extensão universitária. Petrópolis, RJ: Editora Vozes,1996.

BOTOMÉ, S. P.; KUBO, O. M. Responsabilidade social dos programas de Pósgraduação e formação de novos cientistas e professores de nível superior. Interação em Psicologia, Curitiba, v. 6, n. 1, p. 81-110, 2002.

BURT, R. S. Structural holes: the social structure of competition. Cambridge: Harvard University Press, 1992.

BRENTANI, R. R.; CRUZ, C. H. de B.; SUZIGAN, W.; FURTADO, J. E. de M. P.; GARCIA, R. de C. Indicadores de ciência, tecnologia e inovação em São Paulo 2010. São Paulo: FAPESP, 2011

BUFREM, L. S.; SILVA, H. de F. N.; FABIAN, C. L. S. R. e M.; SORRIBAS, T. V. Produção científica em Ciência da Informação. Perspectivas em Ciência da Informação, Belo Horizonte, v. 12, n. 1, p. 38-49, 2007.

CARLOTTO, M. S.; CÂMARA, S. G. Análise da produção científica sobre a Síndrome de Burnout no Brasil. Psico, Rio Grande do Sul, v. 39, n. 2, p. $152-$ 158, 2008.

CRESWELL, J. W. Projeto de pesquisa: métodos qualitativo, quantitativo e misto. 3. ed. Porto Alegre, RS: Artmed, 2010. 296 p.

DANTAS, F. Responsabilidade social e pós-graduação no Brasil: ideias para (avali)ação. Revista Brasileira de Pós-Graduação, Brasília, v. 1, n. 2, p. 160172, 2004.

GLÄNZEL, W. (2004). Bibliometrics as a research field. A course on theory and application of bibliometric indicators. Bélgica: [s.n.], 2003. 
HORTA, J. S. B.; MORAES, M. C. M. de. O sistema CAPES de avaliação da pós-graduação: da área de educação à grande área de ciências humanas.

Revista Brasileira de Educação, Rio de Janeiro, n. 30, 2005.

JANKEVICIUS, J. V. A pesquisa científica e as funções da Universidade. Semina, Londrina, v. 16, n. 2, p. 328-330, jun.1995.

LOURENÇO, C. A. A automação em bibliotecas: análise da produção via Biblioinfo (1986/1994). In: WITTER, G. P. (Org.). Produção científica. Campinas: Átomo, 1997. p. 25-40.

MACIEL, C. O. Práxis estratégicas e imersão social em uma rede de organizações religiosas. 2007. 159f. Dissertação (Mestrado em Administração) - Pós-Graduação em Administração. Universidade Federal do Paraná, Curitiba, PR, Brasil, 2007.

MEADOWS, A. J. A comunicação científica. Brasília: Briquet de Lemos/Livros, 1999.

MOREIRA, J. R.; VILAN FILHO, J. L.; MUELLER, S. P. M. Características e produção científica dos grupos de pesquisa do CNPq/DGP nas áreas de Ciência da Informação e Museologia (1992 - 2012). Perspectivas em Ciência da Informação, Belo Horizonte, v. 20, n. 4, p. 93-106, 2015

MOREIRA, J. R. As áreas de informação no Brasil: um estudo bibliométrico sobre características dos grupos de pesquisa e a produção científica de seus membros. 2017. 354 f. Tese (Doutorado em Ciência da Informação) Programa de pós-graduação em Ciência da Informação, Universidade de Brasília, Brasília, 2017.

MUGNAINI, R.; JANNUZZI, P. de M.; QUONIAM, L. M. Indicadores bibliométricos da produção científica brasileira: uma análise a partir da base Pascal. Ci. Inf., Brasília, v. 33, n. 2, p. 123-131, maio/ago. 2004.

MUGNAINI, R.; CARVALHO, T.; CAMPANATTI-ORTIZ, H. Indicadores de produção científica: uma discussão conceitual. In: POBLACION, D. A.; WITTER, G. P.; SILVA, J. F. M. (orgs.). Comunicação e produção científica: contexto e avaliação. São Paulo: Angellara, 2006. p. 313-340.

OLIVEIRA, H. V. de. Fatores influentes na visibilidade internacional da comunicação científica de pesquisadores de instituições da Amazônia brasileira. Rev. Cult. Pará, Belém, v. 15, n. 1, p. 61-141, 2004.

PAYNE, G. PAYNE, J. Key concept in social research. Londres: Sage, 2004. 242p.

ROSSONI, L.; GUARIDO FILHO, E. R. Cooperação entre programas de pósgraduação em administração no Brasil: evidências estruturais em quatro áreas temáticas. RAC, Maringá, v. 13, n. 3, p. 366-390, 2009. 
SALOMÓN, Y. P.; RODRíGUEZ, A. M. Producción científica. Ciencias de la Información, Cuba, v. 38, n. 3, p.33-38, 2007.

SCHMITT, B. D.; BERTOLDI, R.; MAZO, J. Z. Grupos de pesquisa em ciências da saúde e o uso da história oral. Arquivos de Ciências da Saúde, São José do Rio Preto, v. 24, n. 2, p. 9-13, 2017.

SILVA, A. B. O.; MATHEUS, R. F.; PARREIRAS, F. S.; PARREIRAS, T. A. S. Análise de redes sociais como metodologia de apoio para a discussão da interdisciplinaridade na ciência da informação. Ciência da Informação, Brasília, v. 35, n. 1, p. 72-93, 2006.

SILVA, E. L. da; PINHEIRO, L. V. A produção do conhecimento em ciência da informação: uma análise a partir dos artigos científicos publicados na área. Intexto, Porto Alegre, v. 2, n. 19, p. 1-24, 2008.

SILVA, M. C.; CASIMIRO, A. H. T.; DUARTE, E. N. Caracterização dos grupos de pesquisa em inteligência organizacional competitiva. Biblionline, João Pessoa, v. 12, n. 1, p. 14-25, 2016

SILVEIRA, J. P. B. A produção científica em periódicos institucionais: um estudo da revista Biblios. Encontros Bibli: revista eletrônica de biblioteconomia e ciência da informação, Santa Catarina, v. 17, n. 33, p. 116133, 2012.

VERCESI, A. Os desafios da pesquisa no Brasil. Caderno Temático: Suplemento do Jornal da Unicamp, Campinas. n. 12, ano 1, 2002.

VILAN FILHO, J. L.; MUELLER, S. P. M. A colaboração nos artigos brasileiros de informação: o peso das parcerias orientador-orientando. In: Encontro Brasileiro de Bibliometria e Cientometria, 2., São Carlos. Anais... São Carlos: UFSCar, 2010.

VOOS, H. Lotka and information science. Journal of the American Society of Information Science, New York, v. 25, n. 4, p. 270-272, 1974.

WITTER, G. P. Pós-graduação e produção científica: a questão de autoria. Transinformação, Campinas, v. 1, n. 1, p. 29-37, 1989.

WITTER, G. P (org.). Produção Científica. Campinas: Átomo, 1997.

WITTER, G. P. Análise de produção científica. Psicologia Escolar e Educacional, Campinas, v. 5, n. 1, 2001.

\section{SCIENTIFIC PRODUCTION IN THE POSTGRADUATE PROGRAMS IN INFORMATION AREAS IN BRAZIL}

\section{ABSTRACT}




\begin{abstract}
Understanding the scientific productivity of Postgraduate Programs in the country can promote knowledge about different areas of knowledge. In the case of information areas in Brazil, this panorama can support strategies, decision-making processes, in addition to highlighting research activities, in order to subsidize policies to promote scientific research. However, there are still spaces for studies on how they behave, or what are the characteristics of the scientific production of the information areas in Brazil in different constructs, such as in graduate programs in the respective areas of knowledge, for example. Knowing the factors that induce productivity in postgraduate programs, through its members (researchers and students) can support strategies and policies to foster and maintain research activities. Objective: The objective of this study is to describe the scientific productivity of Brazilian postgraduate programs in the areas of information (Archivology, Library Science, Information Science, Documentation and Museology), represented by its members (researchers and students). Methodology: As a methodological strategy, this study has a quantitative approach, using bibliometrics as a quantitative and statistical technique for measuring the production indexes and dissemination of scientific knowledge. Results: Of the 20 Postgraduate Programs studied, 5 bring together more than half of the total scientific production in the area. The scientific production is divulged, preferably, in channels of communication of complete works in annals of congress. Conclusions: Still in the same context, there is no specific relationship between the volume of researchers and students of the graduate programs and the volume of scientific production.
\end{abstract}

Descriptors: Areas of information in Brazil. Scientific production. Scientific productivity. Postgraduate program.

\title{
PRODUCCIÓN CIENTÍFICA EN PROGRAMAS DE POSGRADO EN ZONAS DE INFORMACIÓN EN BRASIL
}

\begin{abstract}
RESUMEN
Introducción: Comprender la productividad científica de los programas de posgrado en el país puede promover el conocimiento sobre diferentes áreas del conocimiento. En el caso de las áreas de información en Brasil, este panorama puede apoyar estrategias, procesos de toma de decisiones, además de destacar las actividades de investigación, con el fin de subsidiar políticas de promoción de la investigación científica. Sin embargo, aún existen espacios para estudios sobre cómo se comportan, o cuáles son las características de la producción científica de estas áreas en diferentes constructos, como en los programas de posgrado de las respectivas áreas de conocimiento, por ejemplo. Conocer los factores que inducen la productividad en los programas de posgrado a través de sus miembros (investigadores y estudiantes) puede apoyar estrategias y políticas para promover y mantener las actividades de investigación. Objetivo: El objetivo de este estudio es, por tanto, describir la productividad científica de los programas de posgrado brasileños en las áreas de información (Archivología, Bibliotecología, Ciencias de la Información, Documentación y Museología), representados por sus miembros (investigadores y estudiantes). Metodología: Como estrategia metodológica, este estudio tiene un enfoque cuantitativo, utilizando la bibliometría como técnica cuantitativa y estadística para medir índices de producción y difundir el conocimiento científico. Resultados: De los 20 Posgrados estudiados, 5 reúnen más de la mitad de la producción científica total del área. La producción científica se difunde, preferentemente, a través de canales de comunicación de trabajos completos en actas de congresos. Conclusión: Aún en el mismo contexto, no existe
\end{abstract}


una relación específica entre el volumen de investigadores y estudiantes en programas de posgrado y el volumen de producción científica.

Descriptores: Zonas de información en Brasil. Producción científica. Productividad científica. Programa de posgrado.

Recebido em: 02.04.2020.

Aceito em: 21.11.2020 